\title{
Tendão quadricipital: Opção na reconstrução ligamentar do joelho
}

\section{Quadricipital Tendon: Option in Knee Ligament Reconstruction}

\author{
Edmar Stieven Filho ${ }^{1}$ Maisa Sayuri Namba ${ }^{2}$ Isabela do Prado Nascimento ${ }^{3}(0)$ \\ Fernando Martins Rosa ${ }^{4}$ Mario Massatomo Namba ${ }^{5}$
}

${ }^{1}$ Departamento de Ortopedia e Traumatologia, Universidade Federal

Endereço para correspondência Edmar Stieven, Professor Doutor de do Paraná, Curitiba, PR, Brasil

2 Departamento de Ortopedia e Traumatologia, Complexo Hospitalar Ortopedia e Traumatologia - Universidade Federal do Paraná, do Trabalhador, Curitiba, PR, Brasil

${ }^{3}$ Departamento de Medicina, Universidade Federal do Paraná, Departamento de Cirurgia - Ortopedia e Traumatologia, Rua Genera Carneiro, 181. Alto da Glória, Curitiba, Paraná, 80060-900, Brasil Curitiba, PR, Brasil (e-mail: filho2000@gmail.com).

${ }^{4}$ Departamento de Cirurgia, Universidade Federal do Paraná, Curitiba, PR, Brasil

${ }^{5}$ Hospital de Clínicas, Universidade Federal do Paraná, Curitiba, PR, Brasil Rev Bras Ortop 2022;57(1):180-184.

\section{Resumo \\ Palavras-chave \\ - enxertos \\ - ortopedia \\ - ligamento cruzado anterior}
Abstract

\section{Keywords}
- grafts
- orthopedics
- anterior cruciate ligament

\begin{abstract}
Ainda que seja um tendão versátil, apenas $1 \%$ dos cirurgiões optam por utilizar o tendão quadricipital como enxerto na reconstrução do ligamento cruzado anterior (LCA). O presente artigo tem o objetivo de descrever uma técnica de retirada do enxerto quadricipital na qual a sua porção mais profunda é mantida. A técnica consiste em uma abordagem na qual a primeira incisão é feita na porção medial do tendão quadricipital para evitar que ele fique muito curto. Isso acontece devido ao seu desenho triangular. A técnica também aborda a profundidade e a identificação das três camadas do tendão quadricipital para que seja possível preservar sua porção mais profunda. Esta conduta tem o objetivo de uma maior preservação do aparelho extensor e de não haver comunicação com o meio articular, evitando extravasamento de líquido tanto no trans- quanto no pós-operatório.
\end{abstract}

Although it is a versatile tendon, only $1 \%$ of surgeons choose to use the quadricipital tendon as a graft in anterior cruciate ligament $(\mathrm{ACL})$ reconstruction. The present article aims to describe a quadricipital graft removal technique in which its deepest part is maintained. The technique consists of an approach in which the first incision is made in the medial part of the quadricipital tendon to prevent it from getting too short. This is due to its triangular design. The technique also addresses the depth and identification of the three layers of the quadricipital tendon so that it is possible to preserve its deepest part. This approach aims to preserve the extensor apparatus and to not communicate it with the joint environment, avoiding fluid extravasation both in the trans and postoperative periods.
Trabalho desenvolvido no Departamento de Cirurgia - Ortopedia e Traumatologia, Universidade Federal do Paraná, Curitiba, PR, Brasil.

recebido

17 de Agosto de 2020

aceito

01 de Dezembro de 2020

Publicado on-line

Agosto 13, 2021
DOI https://doi.org/ 10.1055/s-0041-1726069. ISSN 0102-3616.

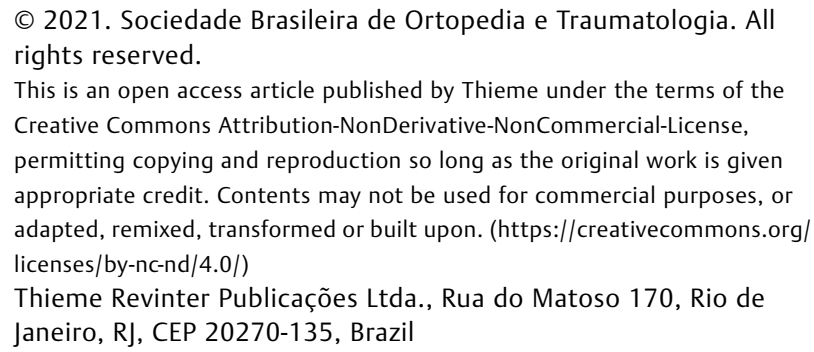

(C) 2021. Sociedade Brasileira de Ortopedia e Traumatologia. All rights reserved.

This is an open access article published by Thieme under the terms of the Creative Commons Attribution-NonDerivative-NonCommercial-License, permitting copying and reproduction so long as the original work is given appropriate credit. Contents may not be used for commercial purposes, or adapted, remixed, transformed or built upon. (https://creativecommons.org/ licenses/by-nc-nd/4.0/) Thieme Revinter Publicações Ltda., Rua do Matoso 170, Rio de Janeiro, RJ, CEP 20270-135, Brazil 


\section{Introdução}

Devido a sua alta incidência na população economicamente ativa, há grande interesse nos avanços de técnicas cirúrgicas de reconstrução do ligamento cruzado anterior(LCA), inclusive em relação aos enxertos utilizados. ${ }^{1}$ Apesar dos avanços, estima-se que $\sim 25 \%$ dos pacientes evoluem com falha nos 10 anos seguintes à cirurgia primária, seja por ruptura do enxerto ou por falha clínica; portanto, muitos destes são submetidos a nova cirurgia, necessitando um segundo enxerto. ${ }^{2,3}$

Considerado por alguns autores como o "enxerto esquecido", o tendão do músculo quadríceps é versátil e pode ser usado com ou sem plugue ósseo na reconstrução ligamentar. ${ }^{4}$ As vantagens incluem resposta clínica favorável e baixa morbidade do sítio doador, principalmente quando é retirado sem o plugue ósseo. Podem ocorrer complicações, como extravasamento de líquido durante a artroscopia, gap muscular, retração do reto femoral, deformidade estética e hematoma no local do enxerto. ${ }^{5}$

Ainda que seja um tendão versátil, apenas $1 \%$ dos cirurgiões optam por utilizar o tendão quadricipital como enxerto na reconstrução do LCA em decorrência da não familiaridade com as técnicas de retirada do enxerto e falta de instrumentação específica. ${ }^{4,5}$

O presente artigo tem o objetivo de descrever uma técnica de retirada do enxerto quadricipital na qual a camada mais profunda, composta pelo vasto intermédio, é mantida.

\section{Descrição Técnica}

O presente estudo foi aprovado pelo Comitê de Ética em Pesquisa através do parecer 35471420.2.0000.5225.

\section{Técnica Cirúrgica}

Passo 1: Preparação e Posicionamento do Paciente O paciente é posicionado em decúbito dorsal, geralmente sob raquianestesia. 0 joelho é posicionado a $90^{\circ}$. Após o

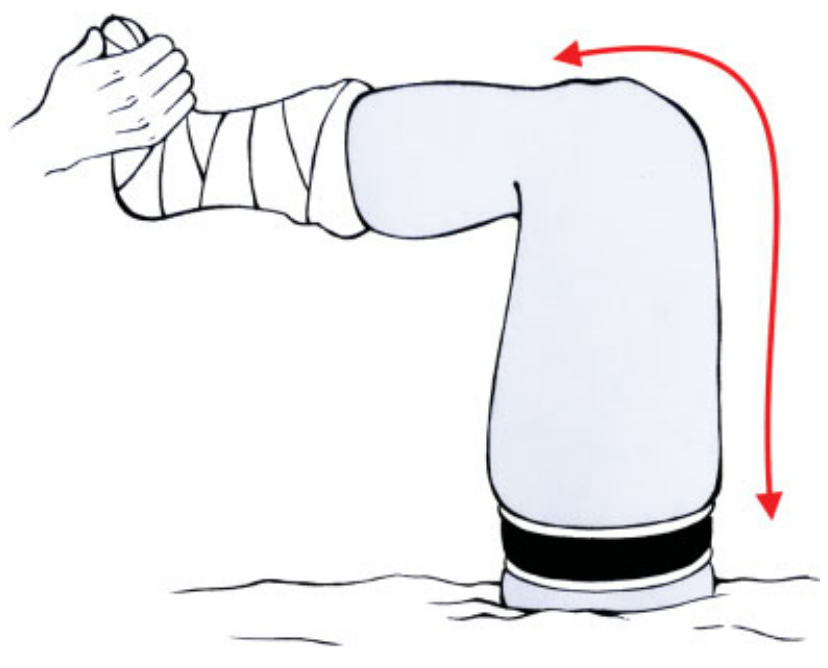

Fig. 1 Posição de $90^{\circ}$ do joelho.

esvaziamento do membro com bandagem de Esmarch, o garrote é insuflado (-Figura 1).

\section{Passo 2: Acesso e Dissecção Subcutâneo}

O centro da patela é utilizado como referência no acesso da pele, o qual é iniciado do centro em direção proximal, de aproximadamente entre 4 e $5 \mathrm{~cm}$. A dissecção de partes moles e do tecido subcutâneo é realizada com o auxílio de bisturi e de uma rugina.

\section{Passo 3: Retirada do Enxerto}

Após o acesso, inicia-se a incisão no enxerto pela porção medial, a entre 3 a $5 \mathrm{~mm}$ laterais à transição miotendínea do bordo proximal do vasto medial oblíquo até o polo proximal da patela (-Figura 2).

A segunda incisão no tendão é realizada a $10 \mathrm{~mm}$ laterais à primeira incisão, determinando a largura do enxerto.

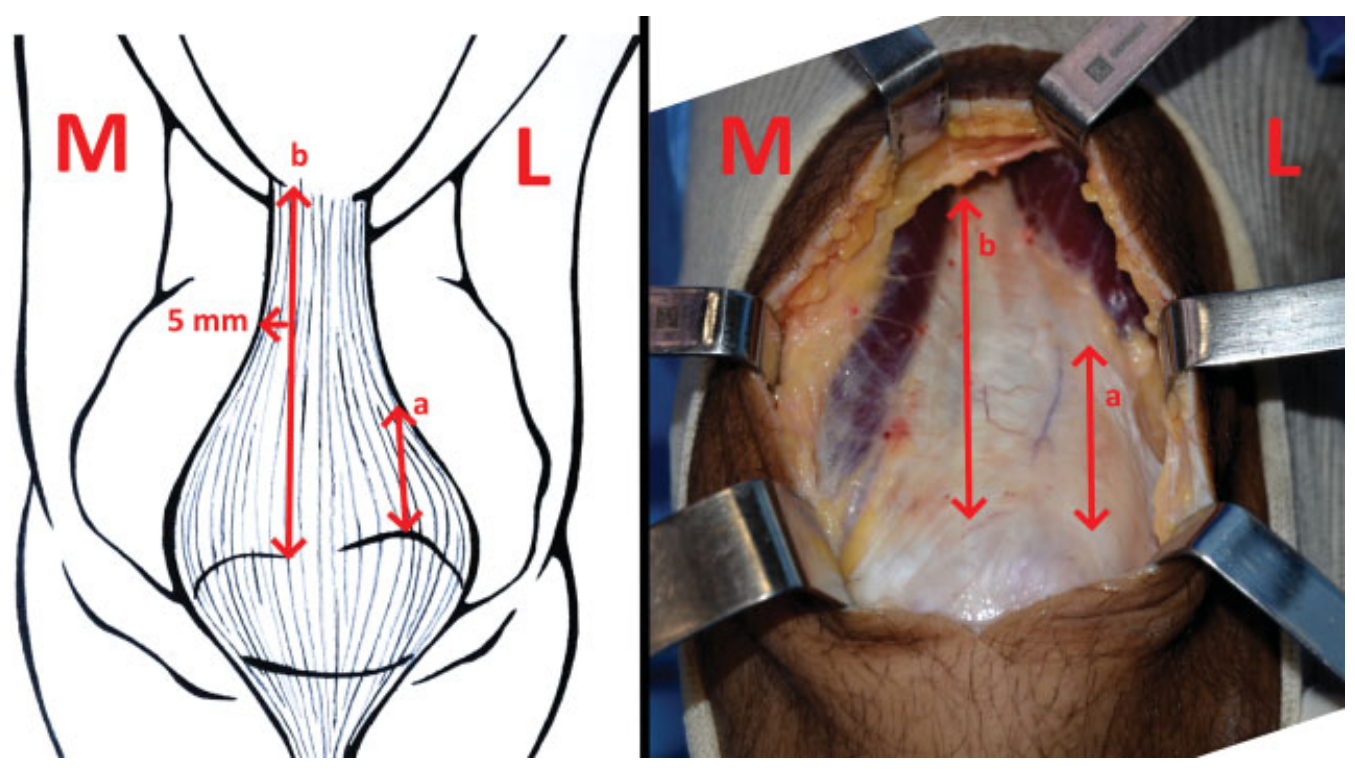

Fig. 2 Primeira incisão no tendão quadricipital, a 3 a 5 mm da miotendínea medial (a. enxerto curto; b. enxerto ideal). M - medial, L - lateral. 
Disseca-se o tendão cuidadosamente, observando suas camadas. A primeira camada é a do reto femoral. Ao dissecá-lo, é possível observar uma lâmina de tecido adiposo separando-o da confluência do vasto medial e lateral. Em seguida, aprofunda-se o bisturi abaixo da gordura e das porções do vasto medial e lateral. Abaixo disso, como terceira camada, está o vasto intermédio, e nesta técnica temos a intenção de preservá-lo. Para auxiliar a medida da profundidade das primeiras duas camadas, a porção mais alta do corte da lâmina do bisturi número 23 pode ser um parâmetro, pois ela possui $\sim 5 \mathrm{~mm}$. Essa é a profundidade aproximada da superfície do tendão quadricipital até o início da terceira camada, o vasto intermédio ( - Figura 3 ).

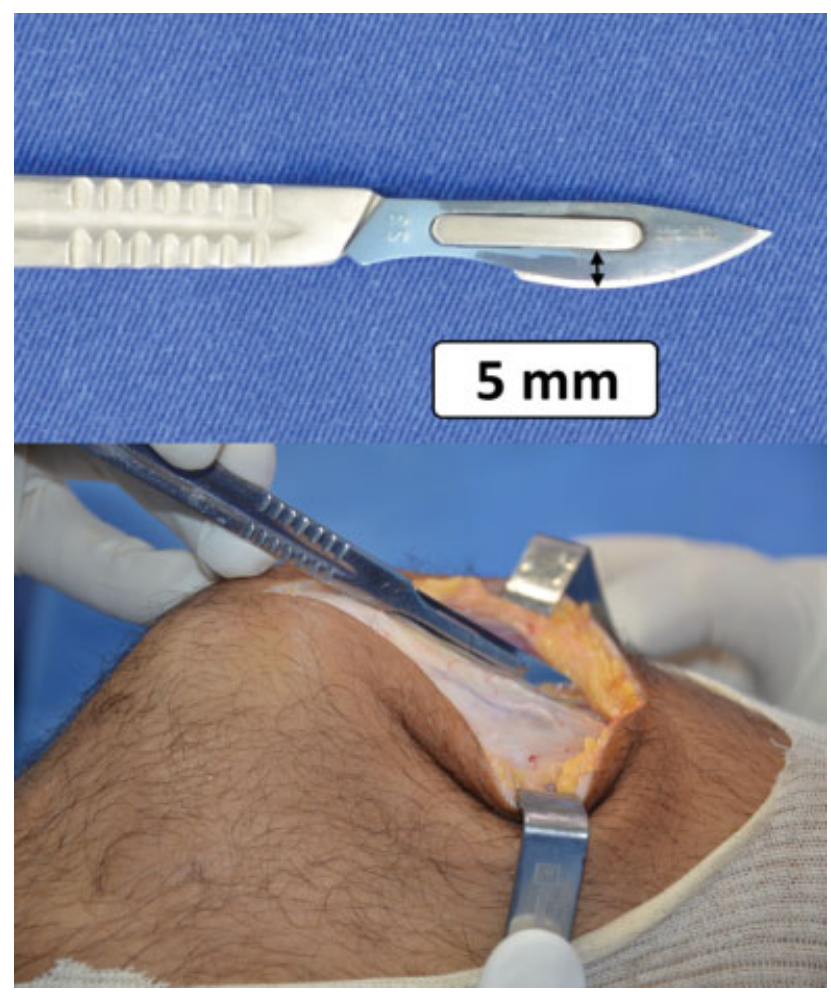

Fig. 3 Bisturi número 23, inserido no tendão quadricipital, com $5 \mathrm{~mm}$ de profundidade.
Após a definição da profundidade, uma pinça hemostática tipo Kelly é posicionada de forma transversal $3 \mathrm{~cm}$ acima do polo proximal da patela, separando o vasto intermédio íntegro do restante do quadríceps. A pinça pode ser forçada proximal e distalmente para ajudar na dissecção ( - Figura 4).

Uma vez definida a camada de corte, a dissecção para proximal e distal pode ser feita de forma romba, forçando a própria pinça Kelly, ou com uma ferramenta de corte. Normalmente, preferimos fazer a dissecção proximal com uma tesoura tipo Metzenbaum e a dissecção até o plugue ósseo com bisturi.

Costumamos utilizar o cautério elétrico para a marcação do plugue ósseo, e temos a preferência por um baguette pequeno ( $15 \mathrm{~mm}$ de comprimento e $10 \mathrm{~mm}$ de largura). Antes de realizar os cortes laterais, o meio do plugue é perfurado com uma broca $3,5 \mathrm{~mm}$ para a passagem da sutura de ancoragem.

Os cortes são realizados com uma serra oscilatória com uma angulação de $45^{\circ}$, de modo a retirar um plugue trapezoidal. Distalmente, o corte é reto em $90^{\circ}$. Após o uso da serra, o plugue do leito é destacado com um osteótomo estreito . Como o tendão quadricipital envolve toda a espessura da patela, é comum que ela continue presa por parte do tendão após a soltura do bloco ósseo (- Figura 5).

Para soltar o enxerto, esta transição é dissecada cuidadosamente para não perfurar o vasto intermédio e expor a articulação, além de evitar a queda do enxerto.

\section{Passo 4: Preparação do Enxerto}

A parte tendinosa do enxerto é preparada com pontos tipo Krackov, com fio de poliéster número dois. O plugue ósseo é preparado aparando arestas para formar um túnel ósseo de menor diâmetro. Pelo furo realizado no plugue ósseo, é passado um fio de poliéster número cinco para tração.

\section{Passo 5: Fechamento}

A inspeção do leito é realizada para verificar se não houve violação da Bursa suprapatelar; caso tenha ocorrido, é realizado o fechamento por meio de uma sutura simples com fio de ácido poliglicólico número um.

Realiza-se o procedimento artroscópico antes do fechamento da pele, pois caso haja extravasamento de líquido, é possível localizar por visualização direta o local de drenagem e fechar o orifício.

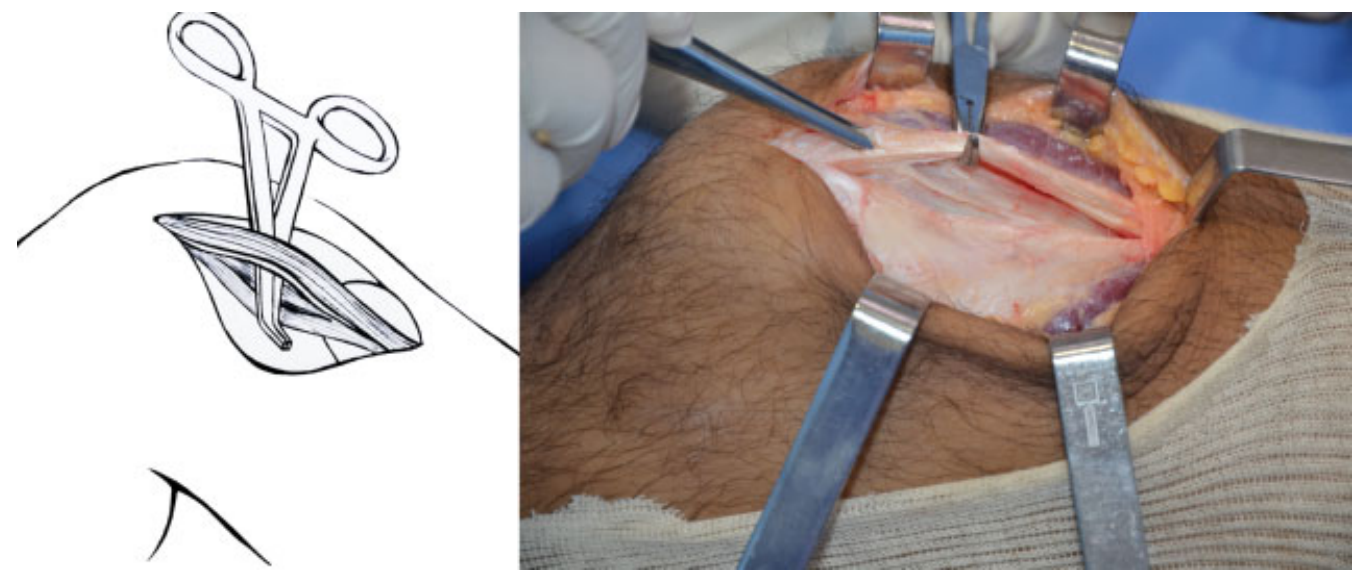

Fig. 4 Tendão quadricipital - o vasto intermédio preservado, enquanto o reto e vastos medial e lateral são reparados pela pinça cirúrgica. 


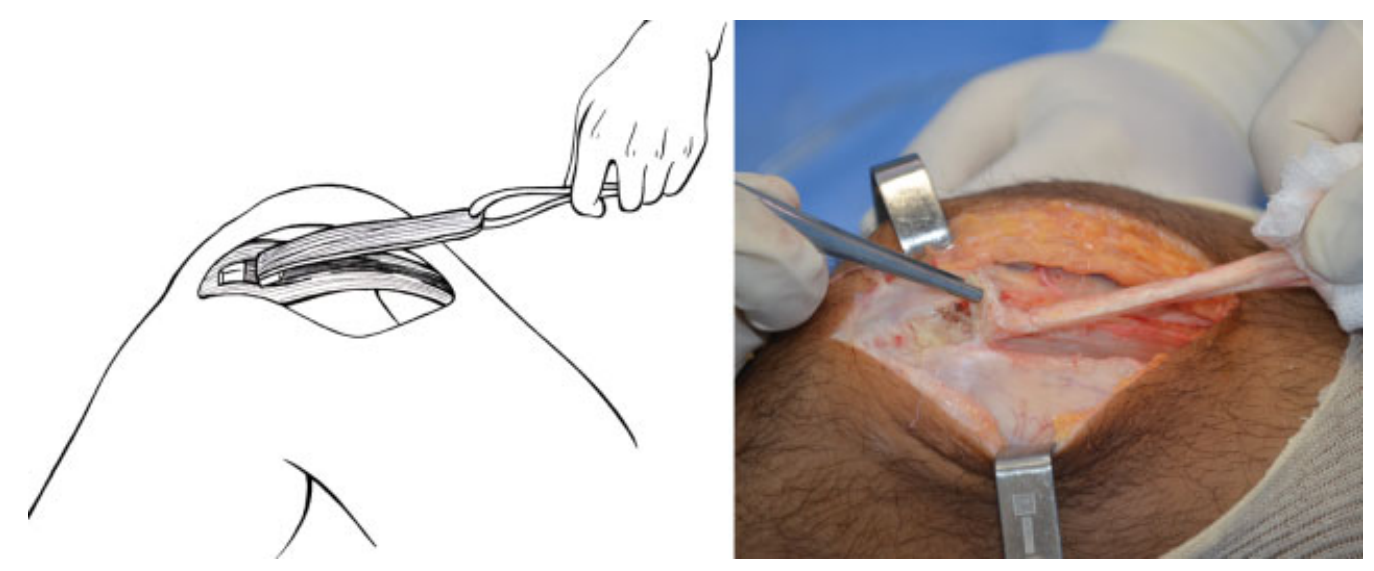

Fig. 5 Enxerto e plugue ósseo do quadríceps. Na foto, observa-se a patela ainda presa no tendão quadricipital.

O garrote é desligado e é realizada uma inspeção e hemostasia.

Não é realizado o preenchimento do defeito da patela e nem a aproximação dos bordos do tendão quadricipital, já que foi preservada a porção mais profunda do vasto intermédio.

\section{Discussão}

Ao preparar e posicionar o paciente, é importante que, ao insuflar o garrote, o joelho esteja fletido em $90^{\circ}$ a fim de manter o músculo quadríceps em sua maior distensão, o que manterá o tendão quadricipital sob menor tensão para a retirada do enxerto. ${ }^{6}$ Para a retirada de enxerto dos tendões flexores, o contrário é realizado: o garrote é insuflado com o joelho estendido (- Figura 1).

Diferente das descrições que iniciam a retirada do enxerto quadricipital pelo bloco ósseo, ${ }^{7}$ julgamos fundamental que a primeira incisão seja no tendão quadricipital. Iniciamos com uma incisão linear, paralela ao tendão, de 3 a $5 \mathrm{~mm}$ laterais à região miotendínea do vasto medial. 0 motivo de insistirmos nesse ponto é que o enxerto quadricipital é triangular e sua porção medial é mais paralela ao fêmur do que a lateral. Assim, temos o cuidado de não realizar a incisão muito lateral, pois isso nos traria um enxerto muito curto (-Figura 2).

Existe uma fina camada de tecido adiposo que separa o reto femoral do vasto intermédio, sendo mais fácil separar o reto femoral da segunda camada do que a segunda da terceira. Como estamos propondo uma técnica na qual haja uma preservação da última camada do tendão quadricipital, é necessário aprofundar a incisão além da divisão do reto femoral com a segunda camada. Assim, aumenta-se a espessura do tendão e preserva-se o vasto intermédio. Este passo nem sempre é simples, pois há uma tendência de pararmos após o reto femoral.

Após a retirada do tendão e do garrote, consideramos importante a inspeção para verificar se há comunicação com o meio articular e a avaliação da hemostasia. Isto é especialmente importante em casos de cirurgias intra-articulares, pois essa comunicação pode causar um grande hematoma no quadríceps no pós-operatório. ${ }^{5}$ Este tipo de hematoma é doloroso e dificulta a reabilitação. Por isso, deixamos a pele e o subcutâneo para serem fechados no final do procedimento, pois caso haja sinais de saída de líquido durante a cirurgia, pode-se fazer o fechamento.

Ferrer et al. ${ }^{8}$ realizaram um estudo por tomografia computadorizada (TC) para verificar o padrão das patelas que sofreram fratura após a retirada do enxerto do tendão quadricipital. Fraturas são mais comuns em casos nos quais o bloco foi retirado mais lateralmente e quando excede $30 \%$ da espessura da patela. Isto reforça nosso pensamento em manter a primeira incisão próxima ao vasto medial. Também acreditamos que um baguette pequeno é o suficiente para a realização da maioria dos procedimentos que requerem este tipo de enxerto.

\section{Considerações Finais}

O início da incisão $5 \mathrm{~mm}$ laterais à região miotendínea medial pode evitar a retirada de um enxerto curto.

É possível retirar duas das três camadas do enxerto quadricipital preservando parte do aparelho extensor e obter um enxerto adequado.

\section{Suporte Financeiro}

Não houve suporte financeiro de fontes públicas, comerciais, ou sem fins lucrativos.

Conflito de Interesses

Os autores declaram não haver conflito de interesses.

\section{Referências}

1 Barié A, Köpf M, Jaber A, et al. Long-term follow-up after anterior cruciate ligament reconstruction using a press-fit quadriceps tendon-patellar bone autograft. BMC Musculoskelet Disord 2018;19(01):368

2 Condello V, Zdanowicz U, Di Matteo B, et al. Allograft tendons are a safe and effective option for revision ACL reconstruction: a clinical review. Knee Surg Sports Traumatol Arthrosc 2019;27 (06):1771-1781

3 Crawford SN, Waterman BR, Lubowitz JH. Long-term failure of anterior cruciate ligament reconstruction. Arthroscopy 2013;29 (09):1566-1571 
184 Tendão quadricipital: opção na reconstrução ligamentar do Joelho Stieven Filho et al.

4 van Eck CF, Illingworth KD, Fu FH. Quadriceps tendon: the forgotten graft. Arthroscopy 2010;26(04):441-442, author reply 442-443

5 Slone HS, Romine SE, Premkumar A, Xerogeanes JW. Quadriceps tendon autograft for anterior cruciate ligament reconstruction: a comprehensive review of current literature and systematic review of clinical results. Arthroscopy 2015;31(03):541-554

6 DeAngelis JP, Fulkerson JP. Quadriceps tendon-a reliable alternative for reconstruction of the anterior cruciate ligament. Clin Sports Med 2007;26(04):587-596
7 Fulkerson JP, Langeland R. An alternative cruciate reconstruction graft: the central quadriceps tendon. Arthroscopy 1995;11(02): 252-254

8 Ferrer GA, Miller RM, Murawski CD, et al. Quantitative analysis of the patella following the harvest of a quadriceps tendon autograft with a bone block. Knee Surg Sports Traumatol Arthrosc 2016;24 (09):2899-2905 